\title{
Nonalcoholic Fatty Liver Disease and Cardiovascular Disease Risk
}

\author{
Roger K. Schindhelm, BSc, MD, MEpi, \\ Michaela Diamant, MD, PhD, and Robert J. Heine, MD, PhD, FRCP
}

\author{
Corresponding author \\ Robert J. Heine, MD, PhD, FRCP \\ Department of Endocrinology/Diabetes Center, VU University Medical \\ Center, PO Box 7057, 1007 MB Amsterdam, The Netherlands. \\ E-mail: rj.heine@vumc.nl
}

Current Diabetes Reports 2007, 7:181-187

Current Medicine Group LLC ISSN 1534-4827

Copyright $@ 2007$ by Current Medicine Group LLC

Nonalcoholic fatty liver disease (NAFLD) is prevalent in people with the metabolic syndrome and type 2 diabetes. Evidence is now accumulating that NAFLD is associated with obesity and diabetes and may serve as a predictor of cardiovascular disease. Although at present, treatment of the individual risk factors pertinent to NAFLD is advocated, novel therapies are emerging that may target steatosis and/or inflammation, thus ameliorating the overall cardiovascular disease risk. Long-term outcome studies need to establish whether treatment of NAFLD (and in particular which therapy) will affect the long-term outcome.

\section{Introduction}

In the past decade, nonalcoholic fatty liver disease (NAFLD) has gained much interest. NAFLD, which is characterized by a wide spectrum of liver disease ranging from liver steatosis to the more severe nonalcoholic steatohepatitis (NASH), resembles alcohol-induced liver disease. By definition, NAFLD develops in subjects who are not heavy alcohol consumers and who have negative tests for viral and autoimmune liver diseases. It usually has a benign clinical course, but it may progress to NASH, fibrosis, cirrhosis, and rarely to hepatocellular carcinoma [1,2]. The term NASH was introduced by Ludwig et al. [3] in 1980, who reported 20 moderately obese patients with liver biopsy changes resembling alcohol-induced hepatitis, although none of these patients reported a history of alcohol abuse. In recent years, NAFLD has gained appreciation as a pathogenic factor of insulin resistance and type 2 diabetes mellitus (T2DM). Furthermore, several studies showed an association between NAFLD and features of the metabolic syndrome, including dyslipidemia and (visceral) obesity, stressing the association with insulin resistance as an important feature of NAFLD. Currently, NAFLD is considered by some authors to be the hepatic component of the metabolic syndrome $[4,5]$ and evidence is accumulating that patients with NAFLD have an increased risk for developing cardiovascular disease (CVD).

\section{Epidemiology of NAFLD}

NAFLD is a common condition and may be the most prevalent liver disorder; however, the true incidence and prevalence in the general population are not known. To date, the prevalence of NAFLD has been estimated in selected patient populations or in studies that are to some extent population based. In the NHANES III (third National Health and Nutrition Examination Survey), a population-based sample of over 15,000 subjects, the prevalence using abnormal (ie, above the upper limit of "normal") values of alanine aminotransferase (ALT) and/or aspartate aminotransferase (AST) as markers of NAFLD was estimated to be $7.8 \%$, of which $31 \%$ had an identifiable cause, which would yield a prevalence of NAFLD of $5.4 \%$. However, the reported prevalences are heavily dependent on the chosen cutoff points for ALT. A study in Northern Italy using ultrasound identified fatty liver in $16 \%$ of lean nondrinkers and a prevalence of $76 \%$ in obese nondrinkers [6]. An ultrasound screening study in a Japanese general population found a prevalence of $12 \%$; however, this study did not exclude heavy drinkers, which might have overestimated the prevalence of NAFLD [7]. Overall, in the general population, the estimates of NAFLD range between $3 \%$ to $36 \%$, but with most estimates in the lower range. In obese patients and in patients with T2DM, the prevalence of NAFLD is much higher and may be up to $90 \%$ [8]. However, comparisons of the reported prevalences of NAFLD are largely hampered by the use of different definitions in greatly differing study populations.

\section{Diagnosis of NAFLD}

The majority of patients with NAFLD are asymptomatic, but some may complain of fatigue and right upper quadrant abdominal fullness or even pain. Laboratory analyses performed in patients with complaints or at health checkups show moderately elevated levels of ALT and AST, with an 
AST-to-ALT ratio usually less than 1 . The other enzymes, $\gamma$-glutamyl transferase and alkaline phosphatase, may also be elevated. Noninvasive imaging techniques, such as ultrasonography and CT, may assist in the diagnosis of fatty liver disease. Ultrasound examination of the liver has a sensitivity and specificity of $80 \%$ to $90 \%$ in identifying fatty liver infiltrates, compared with liver biopsies [9]. CT has a higher sensitivity, but is more expensive than ultrasonography. In contrast to liver biopsies, these imaging techniques cannot distinguish steatosis from steatohepatitis. However, the role of liver biopsy, which is regarded as the "gold standard," is controversial because of the elaborate and invasive nature and associated risks of this technique, and the general benign clinical course of NAFLD. In addition, no consensus exists on the standardization of diagnostic criteria based on liver histology, although a number of scoring systems for NAFLD have been introduced [10••]. Only patients who are suspected to have an advanced stage of the disease, based on clinical judgment, ultrasound, and biochemistry, should be considered for liver biopsy. For example, in a patient with T2DM, morbid obesity, advanced age, or an AST-to-ALTratio greater that 1 , a biopsy may be considered. [11]. The American Gastroenterological Association (AGA) recommends that in patients with suspected NAFLD a detailed history on alcohol intake should be taken to exclude the possibility of alcohol-induced hepatic steatosis and steatohepatitis. The initial laboratory assessment should include ALT, AST, alkaline phosphatase, serum bilirubin and albumin levels, prothrombin time, and serologic tests for viral hepatitis infection. An imaging study should be performed when other causes of liver disease have been excluded. The decision to perform a liver biopsy may depend on the specific clinical circumstances of individual patients in addition to the risks and benefits of performing a liver biopsy and should always, as stated by the AGA, include the patient in the decision making process [12].

In epidemiologic studies, ALT has been used as a marker for liver fat accumulation. Studies assessing the specificity and sensitivity of ALT as a marker of NAFLD are limited $[13,14]$ and applying the laboratory defined cutoff value (ie, upper limit of the laboratory reference range) may underestimate the prevalence of NAFLD [14]. Prati et al. [15] have suggested new cutoff values for ALT in order to facilitate the identification of subjects with NAFLD. However, because cutoff values largely depend on the assay used, this proposal has been questioned [16]. One study has assessed the correlation of ALT with proton spectroscopy MRI; it found a modest, but significant correlation $(r=0.5)$ [17]. As is the case for clinical diagnosis, a detailed history of alcohol intake is mandatory to exclude those patients with ALT elevation caused by alcohol abuse and to address alcohol intake as a possible confounder or effect modifier. Viral and autoimmune liver disease and hepatotoxic medication should be considered as well. With these limitations in mind, ALT may be an acceptable marker for hepatic steatosis in epidemiologic studies.

\section{NAFLD in Relation to the Metabolic} Syndrome and Type 2 Diabetes

The putative role of the liver in the pathogenesis of T2DM has gained much interest. Several cross-sectional studies have demonstrated that NAFLD is related to features of the metabolic syndrome and T2DM [18-20]. In the analysis of the NHANES III, up to $31 \%$ of the elevated aminotransferase activity could be explained by high alcohol consumption, hepatitis B or C infection, and/or high transferrin saturation, whereas in the remaining $69 \%$, the elevated ALT activity was significantly associated with higher body mass index (BMI), waist circumference, triglycerides, fasting insulin, and lower high-density lipoprotein (HDL) cholesterol [18]. Several studies have addressed the prospective relation of ALT and the metabolic syndrome and T2DM. Nakanishi et al. [21] found that ALT was associated with future risk of metabolic syndrome in middle-aged Japanese men, but used BMI instead of waist circumference to define the metabolic syndrome. Hanley et al. [22] studied the relationship of four different liver enzymes (including ALT) with the development of the metabolic syndrome in a multi-ethnic cohort. They demonstrated that ALT was associated with an increased risk of incident metabolic syndrome. In the Hoorn Study, a population-based cohort study among elderly white men and women, we found that ALT was associated with the development of the metabolic syndrome after 6 years of follow-up [23].

In patients with T2DM, elevated serum ALT enzyme activity is more frequently observed than in the general population [24,25]. In addition, some [26-30], but not all studies [21,31-33] have demonstrated independent and significant associations of ALT with future T2DM. In 1988, Ohlson et al. [26] demonstrated that baseline ALT was a predictor of incident T2DM after 13.5 years of follow-up in a cohort of 766 Swedish men, with a significant fourfold risk for those men in the upper quintile compared with those in the lower quintile. In the final multivariate model, ALT was a predictor of incident T2DM in 451 Pima Indians after an average of 6.9 years, after adjustment for fasting blood glucose levels, BMI, bilirubin, systolic blood pressure, uric acid, and a family history of diabetes [26]. Vozarova et al. [27] found that higher ALT (upper compared with lower decile) was a significant and independent predictor of T2DM with a twofold increase risk, after adjustment for age, sex, body fat, insulin sensitivity, and acute insulin response. Other studies, mainly performed in men, confirmed these earlier studies [28,29], whereas recent population-based studies could not demonstrate independent associations of ALT and future risk of T2DM $[32,33]$. The observed association between ALT and incident T2DM in the mentioned studies may be explained by the fact that they were performed in high-risk populations, which may not be representative of the general population. Overall, these studies show that patients with NAFLD are at increased risk for T2DM and the metabolic syndrome, suggesting that the increased CVD risk may be mediated via components of the metabolic 


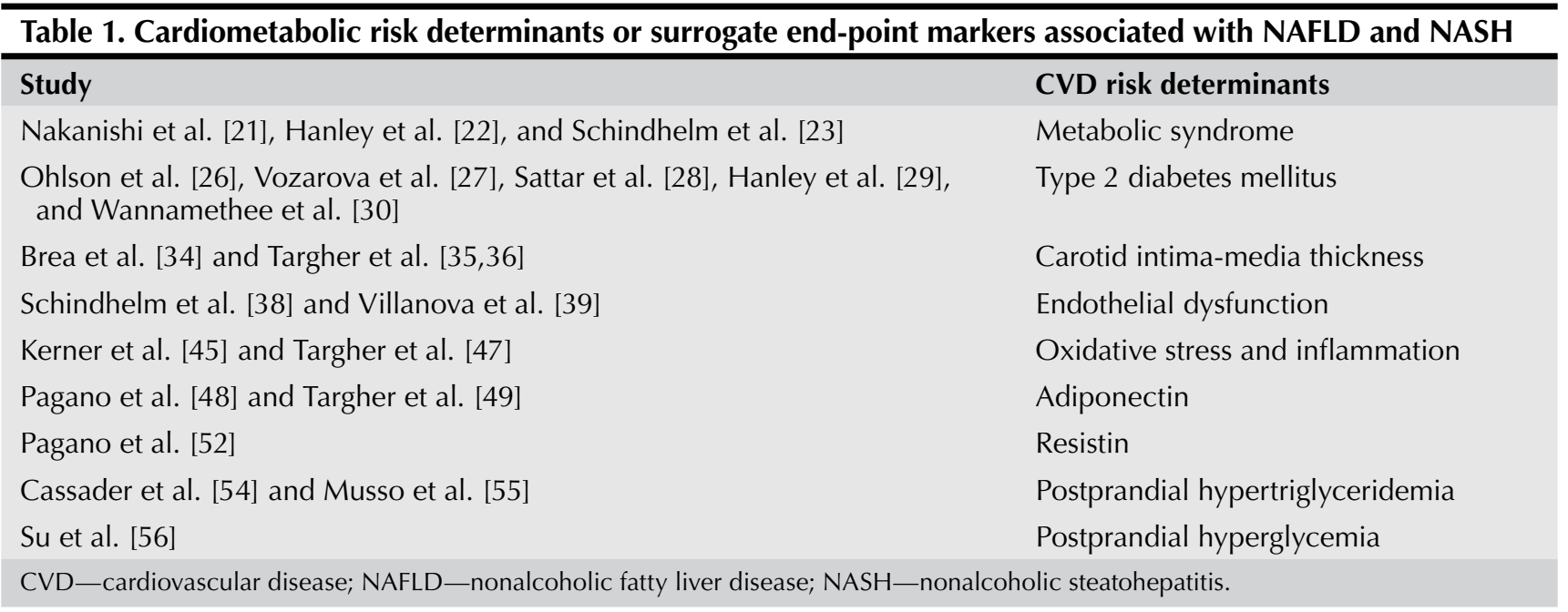

syndrome and T2DM. Because the pathophysiology linking NAFLD with either the metabolic syndrome and/or diabetes has not been clarified, it is difficult to make the distinction between confounding and mediating variables in the epidemiologic analyses.

\section{NAFLD and Increased CVD Risk}

Several cross-sectional studies have demonstrated an increase in carotid artery intima-media thickness (CIMT) in patients with NAFLD [34-36]. However, in these studies diagnosis of NAFLD was based on evaluation of liver enzymes or on ultrasound evaluation but not confirmed by liver biopsy, which is regarded as the "gold standard" in the diagnosis of NAFLD [10••]. In a recent study,

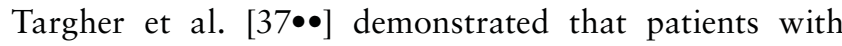
biopsy-proven NAFLD have a significantly higher CIMT compared with age-, sex-, and BMI-matched healthy control subjects. Moreover, they demonstrated that the histologically assessed NAFLD predicted CIMT, independent of classical risk factors, including insulin resistance and components of the metabolic syndrome.

Previously, in patients with well-controlled T2DM, we have shown that slightly elevated ALT, as a surrogate marker of NAFLD, is associated with a decreased brachial artery flow-mediated vasodilatation and an impaired whole-body insulin sensitivity [38]. In line with the observation in patients with T2DM, it was shown that nondiabetic patients with NAFLD have a decreased brachial artery flow-mediated vasodilatation compared with matched healthy control subjects, independent of other risk factors, including insulin resistance and components of the metabolic syndrome [39]. Ioannou et al. [40] studied the association of ALT and the calculated 10 -year risk of coronary heart disease as estimated with the Framingham risk score. Subjects with elevated ALT values had a significantly higher Framingham risk score compared to those with normal ALT values, indicating a higher CVD risk in patients with NAFLD [40].
Only a limited number of studies have addressed the prospective relation of ALT with CVD and mortality. In the Hoorn Study, a population-based cohort of white men and women aged 50 to 75 years, we studied the association of ALT at baseline with all-cause mortality and incident cardiovascular and coronary heart disease events [41] and found a significant association of ALT with coronary heart disease after adjustment for components of the metabolic syndrome and traditional CVD risk factors. We found no independent associations of ALT with CVD events and all-cause mortality. The latter result was in line with a previous study by Arndt et al. [42], who studied the association of ALT with all-cause mortality in 8043 male construction workers and found no significant association. In contrast, a recent study by Nakamura et al. [43] found a positive association of ALT with all-cause mortality in Japanese men and women, but only for those with a BMI below the median $\left(22.7 \mathrm{~kg} / \mathrm{m}^{2}\right)$.

\section{Mechanisms Linking NAFLD to Increased CVD Risk}

The increased CVD risk associated with NAFLD might be explained by the close relation of NAFLD with components of the metabolic syndrome and T2DM; however, recent studies support the notion that NAFLD in itself might contribute to the increased CVD risk (Table 1). However, the mechanisms for this putative relationship are not clear. Several probably highly interrelated factors contribute to the enhanced risk of diabetes and metabolic syndrome in persons with NAFLD (Fig. 1).

\section{Oxidative stress and inflammation}

Hepatic steatosis mediated by insulin resistance is required for subsequent events (of which oxidative stress is an important contributor) that lead to liver injury and disease progression [44]. However, the underlying factors that promote disease progression to cirrhosis are not understood. Enhanced free fatty acid oxidation increases the formation of oxygen 


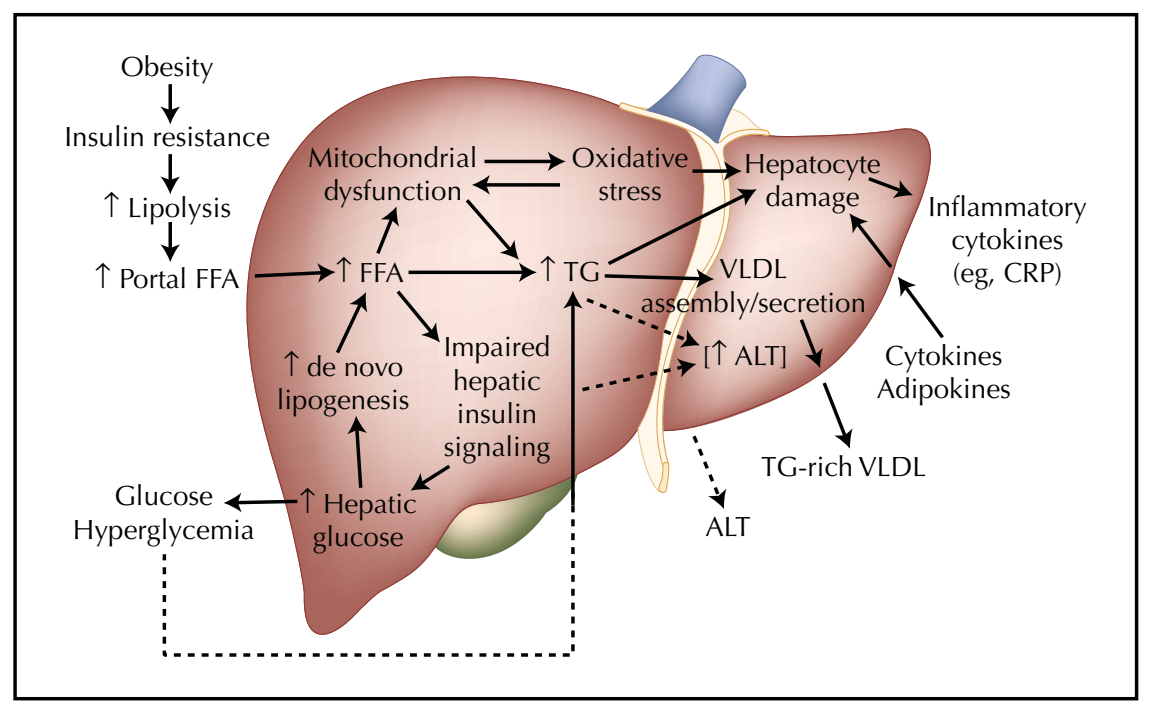

Figure 1. Overview of the mechanisms-increased uptake of free fatty acids (FFAs) by the liver, an increase in de novo lipogenesis, impaired $\beta$ oxidation caused by mitochondrial dysfunction, and an insufficient assembly and secretion of very low density lipoproteins (VLDLs) —involved in the development of nonalcoholic fatty liver disease (NAFLD) and the contribution of NAFLD to cardiovascular risk. ALT_alanine aminotransferase; $\mathrm{CRP}-\mathrm{C}$-reactive protein; TG-triglycerides. radicals, in turn leading to lipid peroxidation, mitochondrial dysfunction, and cell damage with subsequent release of cytokines, including tumor necrosis factor- $\alpha$, interleukin- 6 , and C-reactive protein (CRP). Kerner et al. [45] found an association of ALT with CRP. In contrast, Haukeland et al. [46] studied the role of systemic inflammation in individuals with NAFLD and NASH compared with healthy control subjects. It was demonstrated that interleukin-6, but not CRP, after adjustment for BMI, age, and sex, was elevated in NAFLD compared with control subjects and tumor necrosis factor- $\alpha$ was higher in NASH compared with NAFLD [46]. Of interest, a study by Targher et al. [47] demonstrated that CRP was elevated in individuals with NAFLD, but this association was largely explained by the amount of visceral fat.

\section{Adiponectin, leptin, and resistin}

Decreased adiponectin levels may represent another mechanism linking NAFLD to CVD. Patients with NAFLD have lower levels of adiponectin compared with healthy control subjects, independent of components of the metabolic syndrome $[46,48,49]$. This observation may be relevant because some studies have shown that lower levels of adiponectin are associated with CVD [50]. Leptin is a cytokine hormone mainly produced by adipocytes, which regulates food intake and fat metabolism through actions on the central nervous system. It has been suggested that leptin may act as one of the regulators in progression of NAFLD to NASH by upregulation of transforming growth factor- $\beta$ [51]. However, a recent study found no association of leptin with liver disease severity [52]. Resistin is a protein expressed in adipose tissue and related to insulin resistance in mice [53]. Recently, a relationship of resistin, NAFLD, and NASH was demonstrated in humans, with higher plasma resistin levels in individuals with NASH [52].

\section{Postprandial dysmetabolism}

Studies comparing the postprandial response of triglycerides and free fatty acids to a fat-rich meal in nondiabetic subjects with biopsy-proven NASH to control subjects showed that patients with NASH had significantly higher postprandial triglyceride levels than healthy control subjects [54,55]. Another study demonstrated that patients with ultrasound-diagnosed NAFLD with an abnormal ALT and/or AST had higher glucose levels after a 75-g oral glucose tolerance test than those with normal ALT and/or AST [56]. Toledo et al. [57••] observed that in obese patients with T2DM, increased hepatic steatosis, as quantified by CT scanning, was positively correlated with serum triglycerides and inversely with HDL cholesterol. No difference in low-density lipoprotein (LDL) cholesterol and apolipoprotein B100 was observed relative to the degree of hepatic steatosis. However, the LDL particle size was smaller in the group with severe hepatic steatosis. These observations indicate that hepatic steatosis may contribute to the increased CVD risk in these patients by increasing triglyceride enrichment of very low density lipoprotein (VLDL) particles, lowering HDL cholesterol and by increasing small, dense LDL particles. The relationship of hepatic steatosis with serum triglycerides was stronger in subjects with a minor degree of hepatic steatosis and weaker in those with a more severe degree of hepatic steatosis, which may, as the authors suggested, indicate that that the incorporation of triglycerides into

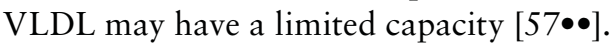

\section{Management of NAFLD-associated}

Cardiometabolic Abnormalities or NAFLD?

Lifestyle interventions, including diets and exercise, leading to weight loss and concomitant improvement of the CVD risk profile, have been shown to lower liver fat content $[58,59]$. These interventions may improve insulin sensitivity and/or reduce oxidative stress, both important pathophysiologic factors in the pathogenesis of NAFLD and CVD. Although these lifestyle interventions seem promising in the short term, long-term effects have not yet 
been established and might not be sufficient in patients with multiple cardiometabolic risk factors, such as persons with the metabolic syndrome and/or T2DM.

Several pharmacologic agents, including metformin and peroxisome proliferator-activated receptor- $\gamma$ (PPAR- $\gamma$ ) agonists, have been used in the treatment of NAFLD. An open-labeled trial of $20 \mathrm{mg} / \mathrm{kg}$ of metformin for 1 year demonstrated only a transient improvement of liver enzymes [60]. Tiikkainen et al. [61] compared the effect of $2 \mathrm{~g}$ of metformin to $8 \mathrm{mg}$ of the PPAR- $\gamma$ agonist rosiglitazone on liver fat content and hepatic insulin sensitivity in a 16-week double-blind randomized trial in 20 subjects. They found that rosiglitazone decreased liver fat by $51 \%$, whereas no significant decrease was observed in the metformin-treated subjects. In contrast, Bugianesi et al. [62] found that $2 \mathrm{~g}$ of metformin led to more ALT normalization (ie, below the reference range) than either vitamin $\mathrm{E}$ or diet-induced weight reduction in 55 subjects in a 12-month open-labeled randomized trial. Metformin induced a decrease of $50 \%$ in liver fat and a decrease of inflammation and necrosis, using paired liver biopsies [62]. Unfortunately, in this study no paired biopsies were performed in the subjects who were randomized to vitamin $\mathrm{E}$ or the weight reduction intervention; thus, the level of liver histology improvement in the treatment group could not be compared with the effect in the control group. A recently reported 6-month placebo-controlled trial of diet combined with pioglitazone compared to diet with placebo in 55 patients with liver biopsy-proven NAFLD demonstrated a significant decrease in ALT and hepatic fat content (by 54\%) and a significant increase in hepatic insulin sensitivity [63••].

More recently introduced pharmacologic agents, including glucagon-like peptide-1 (GLP-1), receptor agonists, incretin-mimetics known to promote insulin production and secretion, and rimonabant, a cannabinoid receptor-1 (CB1) antagonist, may be of therapeutic value in the treatment of NAFLD and NASH. In ob/ob mice, Ding et al. [64] demonstrated that 60 days of administration of a GLP-1 receptor agonist reduced hepatic steatosis. This reduction in hepatic fat content was not only related to a decrease in body weight, but also to a decrease in regulatory genes of fatty acid synthesis [65]. Recently, Tushuizen et al. [65] reported on a 59-year-old male with T2DM treated with a GLP-1 receptor agonist for 44 weeks. They found a significant decrease in liver fat measured by liver spectroscopy, suggesting that GLP-1 receptor agonists may be a potential treatment modality in patients with NAFLD. However, more studies assessing the effect of GLP-1 receptor agonists on hepatic steatosis are needed to confirm these results and assess the applicability of these agents for the treatment of hepatic steatosis [65]. Rimonabant has been shown to be effective in weight reduction and improvement of lipid profile [66]; however, the effect of rimonabant on lowering of liver fat content and improvement of liver histology beyond weight loss in patients with NAFLD needs to be evaluated. Of interest, a recent study demonstrated that treatment with the CB1 receptor antagonist SR14716A in mice inhibited fibrosis in liver injury models [67], suggesting that treatment with a CB1 receptor antagonist may be a future therapeutic modality in NAFLD and NASH.

\section{Conclusions}

Evidence is now accumulating that NAFLD is associated with cardiovascular risk factors, with markers of subclinical atherosclerosis, and with overt CVD events. This increased risk for CVD necessitates the evaluation and treatment of these patients. Long-term outcome studies need to establish the benefit of treatment of NAFLD on the reduction and prevention of diabetes and CVD risk.

\section{References and Recommended Reading}

Papers of particular interest, published recently, have been highlighted as:

- Of importance

- $\quad$ Of major importance

1. Angulo P: Nonalcoholic fatty liver disease. N Engl J Med 2002, 346:1221-1231.

2. Day CP: Genes or environment to determine alcoholic liver disease and non-alcoholic fatty liver disease. Liver Int 2006, 26:1021-1028.

3. Ludwig J, Viggiano TR, McGill DB, Oh BJ: Nonalcoholic steatohepatitis: Mayo Clinic experiences with a hitherto unnamed disease. Mayo Clin Proc 1980, 55:434-438.

4. Cortez-Pinto H, Camilo ME, Baptista A, et al.: Non-alcoholic fatty liver: another feature of the metabolic syndrome? Clin Nutr 1999, 18:353-358.

5. Marchesini G, Brizi M, Bianchi G, et al.: Nonalcoholic fatty liver disease: a feature of the metabolic syndrome. Diabetes 2001, 50:1844-1850.

6. Bellentani S, Saccoccio G, Masutti F, et al.: Prevalence of and risk factors for hepatic steatosis in Northern Italy. Ann Intern Med 2000, 132:112-117.

7. Nomura K, Yano E, Shinozaki T, Tagawa K: Efficacy and effectiveness of liver screening program to detect fatty liver in the periodic health check-ups. J Occup Health 2004, 46:423-428.

8. Clark JM: The epidemiology of nonalcoholic fatty liver disease in adults. J Clin Gastroenterol 2006, 40:S5-S10.

9. Hultcrantz R, Gabrielsson N: Patients with persistent elevation of aminotransferases: investigation with ultrasonography, radionuclide imaging and liver biopsy. J Intern Med 1993, 233:7-12.

$10 . \bullet$ Hubscher SG: Histological assessment of non-alcoholic fatty liver disease. Histopathology 2006, 49:450-465. This paper gives an excellent overview of the histologic assessment of NAFLD and discusses several scoring systems that have been proposed.

11. Angulo P, Keach JC, Batts KP, Lindor KD: Independent predictors of liver fibrosis in patients with nonalcoholic steatohepatitis. Hepatology 1999, 30:1356-1362.

12. Sanyal AJ: AGA technical review on nonalcoholic fatty liver disease. Gastroenterology 2002, 123:1705-1725.

13. Kunde SS, Lazenby AJ, Clements RH, Abrams GA: Spectrum of NAFLD and diagnostic implications of the proposed new normal range for serum ALT in obese women. Hepatology 2005, 42:650-656. 
14. Zelber-Sagi S, Nitzan-Kaluski D, Halpern Z, Oren R: Prevalence of primary non-alcoholic fatty liver disease in a population-based study and its association with biochemical and anthropometric measures. Liver Int 2006, 26:856-863.

15. Prati D, Taioli E, Zanella A, et al.: Updated definitions of healthy ranges for serum alanine aminotransferase levels. Ann Intern Med 2002, 137:1-10.

16. Ratziu V, Imbert-Bismut F, Messous D, Poynard T: The elusiveness of "normal" ALT in fatty liver. Hepatology 2004, 39:1172.

17. Westerbacka J, Corner A, Tiikkainen M, et al.: Women and men have similar amounts of liver and intra-abdominal fat, despite more subcutaneous fat in women: implications for sex differences in markers of cardiovascular risk. Diabetologia 2004, 47:1360-1369.

18. Clark JM, Brancati FL, Diehl AM: The prevalence and etiology of elevated aminotransferase levels in the United States. Am J Gastroenterol 2003, 98:960-967.

19. Ruhl CE, Everhart JE: Determinants of the association of overweight with elevated serum alanine aminotransferase activity in the United States. Gastroenterology 2003, 124:71-79.

20. Ioannou GN, Weiss NS, Boyko EJ, et al.: Contribution of metabolic factors to alanine aminotransferase activity in persons with other causes of liver disease. Gastroenterology 2005, 128:627-635.

21. Nakanishi N, Suzuki K, Tatara K: Serum gamma-glutamyltransferase and risk of metabolic syndrome and type 2 diabetes in middle-aged Japanese men. Diabetes Care 2004, 27:1427-1432.

22. Hanley AJ, Williams K, Festa A, et al.: Liver markers and development of the metabolic syndrome: the insulin resistance atherosclerosis study. Diabetes 2005, 54:3140-3147.

23. Schindhelm RK, Dekker JM, Nijpels G, et al.: Alanine aminotransferase and the 6-year risk of the metabolic syndrome in Caucasian men and women: the Hoorn Study. Diabet Med 2007, in press.

24. Salmela PI, Sotaniemi EA, Niemi M, Maentausta O: Liver function tests in diabetic patients. Diabetes Care 1984, $7: 248-254$

25. Meltzer AA, Everhart JE: Association between diabetes and elevated serum alanine aminotransferase activity among Mexican Americans. Am J Epidemiol 1997, 146:565-571.

26. Ohlson LO, Larsson B, Bjorntorp P, et al.: Risk factors for type 2 (non-insulin-dependent) diabetes mellitus. Thirteen and one-half years of follow-up of the participants in a study of Swedish men born in 1913. Diabetologia 1988, 31:798-805.

27. Vozarova B, Stefan N, Lindsay RS, et al.: High alanine aminotransferase is associated with decreased hepatic insulin sensitivity and predicts the development of type 2 diabetes. Diabetes 2002, 51:1889-1895.

28. Sattar N, Scherbakova O, Ford I, et al.: Elevated alanine aminotransferase predicts new-onset type 2 diabetes independently of classical risk factors, metabolic syndrome, and C-reactive protein in the west of Scotland coronary prevention study. Diabetes 2004, 53:2855-2860.

29. Hanley AJ, Williams K, Festa A, et al.: Elevations in markers of liver injury and risk of type 2 diabetes: the insulin resistance atherosclerosis study. Diabetes 2004, 53:2623-2632.

30. Wannamethee SG, Shaper AG, Lennon L, Whincup PH: Hepatic enzymes, the metabolic syndrome, and the risk of type 2 diabetes in older men. Diabetes Care 2005, 28:2913-2918.

31. Nannipieri M, Gonzales C, Baldi S, et al.: Liver enzymes, the metabolic syndrome, and incident diabetes: the Mexico City diabetes study. Diabetes Care 2005, 28:1757-1762.

32. Schindhelm RK, Dekker JM, Nijpels G, et al.: No independent association of alanine aminotransferase with risk of future type 2 diabetes in the Hoorn study. Diabetes Care 2005, 28:2812.
33. Andre P, Balkau B, Born C, et al.: Hepatic markers and development of type 2 diabetes in middle aged men and women: a three-year follow-up study. Diabetes Metab 2005, 31:542-550.

34. Brea A, Mosquera D, Martin E, et al.: Nonalcoholic fatty liver disease is associated with carotid atherosclerosis: a case-control study. Arterioscler Thromb Vasc Biol 2005, 25:1045-1050.

35. Targher G, Bertolini L, Padovani R, et al.: Non-alcoholic fatty liver disease is associated with carotid artery wall thickness in diet-controlled type 2 diabetic patients. J Endocrinol Invest 2006, 29:55-60.

36. Targher G, Bertolini L, Padovani R, et al.: Relation of nonalcoholic hepatic steatosis to early carotid atherosclerosis in healthy men: role of visceral fat accumulation. Diabetes Care 2004, 27:2498-2500.

37.•• Targher G, Bertolini L, Padovani R, et al.: Relations between carotid artery wall thickness and liver histology in subjects with nonalcoholic fatty liver disease. Diabetes Care 2006, 29:1325-1330.

This is a very elegant study describing the relation between the severity of NAFLD assessed by liver biopsy and the intima-media thickness as a marker of generalized atherosclerosis in individuals with NAFLD.

38. Schindhelm RK, Diamant M, Bakker SJL, et al.: Liver alanine aminotransferase, insulin resistance and endothelial dysfunction in normotriglyceridaemic subjects with type 2 diabetes mellitus. Eur J Clin Invest 2005, 35:369-374.

39. Villanova N, Moscatiello S, Ramilli S, et al.: Endothelial dysfunction and cardiovascular risk profile in nonalcoholic fatty liver disease. Hepatology 2005, 42:473-480.

40. Ioannou GN, Weiss NS, Boyko EJ, et al.: Elevated serum alanine aminotransferase activity and calculated risk of coronary heart disease in the United States. Hepatology 2006, 43:1145-1151.

41. Schindhelm RK, Dekker JM, Nijpels G, et al.: Alanine aminotransferase predicts coronary heart disease events: a 10-year follow-up of the Hoorn study. Atherosclerosis 2006, May 6; [Epub ahead of print].

42. Arndt V, Brenner H, Rothenbacher D, et al.: Elevated liver enzyme activity in construction workers: prevalence and impact on early retirement and all-cause mortality. Int Arch Occup Environ Health 1998, 71:405-412.

43. Nakamura K, Okamura T, Kanda H, et al.: The value of combining serum alanine aminotransferase levels and body mass index to predict mortality and medical costs: a 10year follow-up study of National Health Insurance in Shiga, Japan. J Epidemiol 2006, 16:15-20.

44. Videla LA, Rodrigo R, Araya J, Poniachik J: Insulin resistance and oxidative stress interdependency in non-alcoholic fatty liver disease. Trends Mol Med 2006, 12:555-558.

45. Kerner A, Avizohar O, Sella R, et al.: Association between elevated liver enzymes and C-reactive protein: possible hepatic contribution to systemic inflammation in the metabolic syndrome. Arterioscler Thromb Vasc Biol 2005, 25:193-197.

46. Haukeland JW, Damas JK, Konopski Z, et al.: Systemic inflammation in nonalcoholic fatty liver disease is characterized by elevated levels of CCL2. J Hepatol 2006, 44:1167-1174.

47. Targher G, Bertolini L, Scala L, et al.: Non-alcoholic hepatic steatosis and its relation to increased plasma biomarkers of inflammation and endothelial dysfunction in non-diabetic men. Role of visceral adipose tissue. Diabet Med 2005, 22:1354-1358.

48. Pagano C, Soardo G, Esposito W, et al.: Plasma adiponectin is decreased in nonalcoholic fatty liver disease. Eur $J$ Endocrinol 2005, 152:113-118.

49. Targher G, Bertolini L, Scala L, et al.: Decreased plasma adiponectin concentrations are closely associated with nonalcoholic hepatic steatosis in obese individuals. Clin Endocrinol (Oxf) 2004, 61:700-703. 
50. Pischon T, Girman CJ, Hotamisligil GS, et al.: Plasma adiponectin levels and risk of myocardial infarction in men. JAMA 2004, 291:1730-1737.

51. Ikejima K, Okumura K, Lang T, et al.: The role of leptin in progression of non-alcoholic fatty liver disease. Hepatol Res 2005, 33:151-154.

52. Pagano C, Soardo G, Pilon C, et al.: Increased serum resistin in nonalcoholic fatty liver disease is related to liver disease severity and not to insulin resistance. J Clin Endocrinol Metab 2006, 91:1081-1086.

53. Muse ED, Obici S, Bhanot S, et al.: Role of resistin in diet-induced hepatic insulin resistance. J Clin Invest 2004, 114:232-239.

54. Cassader M, Gambino R, Musso G, et al.: Postprandial triglyceride-rich lipoprotein metabolism and insulin sensitivity in nonalcoholic steatohepatitis patients. Lipids 2001, 36:1117-1124.

55. Musso G, Gambino R, De Michieli F, et al.: Dietary habits and their relations to insulin resistance and postprandial lipemia in nonalcoholic steatohepatitis. Hepatology 2003, 37:909-916.

56. Su CC, Wang K, Hsia TL, et al.: Association of nonalcoholic fatty liver disease with abnormal aminotransferase and postprandial hyperglycemia. J Clin Gastroenterol 2006, 40:551-554.

57.• Toledo FG, Sniderman AD, Kelley DE: Influence of hepatic steatosis (fatty liver) on severity and composition of dyslipidemia in type 2 diabetes. Diabetes Care 2006, 29:1845-1850.

This study reports on the relation of hepatic steatosis and dyslipidemia. It found that the degree of liver fat accumulation was associated with increased serum triglyceride concentrations and increased HDL cholesterol. No difference in LDL cholesterol was found; however, the LDL particle size was smaller in individuals with severe hepatic steatosis.

58. Ueno T, Sugawara H, Sujaku K, et al.: Therapeutic effects of restricted diet and exercise in obese patients with fatty liver. J Hepatol 1997, 27:103-107.
59. Hickman IJ, Jonsson JR, Prins JB, et al.: Modest weight loss and physical activity in overweight patients with chronic liver disease results in sustained improvements in alanine aminotransferase, fasting insulin, and quality of life. Gut 2004, 53:413-419.

60. Nair S, Diehl AM, Wiseman M, et al.: Metformin in the treatment of non-alcoholic steatohepatitis: a pilot open label trial. Aliment Pharmacol Ther 2004, 20:23-28.

61. Tiikkainen M, Hakkinen AM, Korsheninnikova E, et al.: Effects of rosiglitazone and metformin on liver fat content, hepatic insulin resistance, insulin clearance, and gene expression in adipose tissue in patients with type 2 diabetes. Diabetes 2004, 53:2169-2176.

62. Bugianesi E, Gentilcore E, Manini R, et al.: A randomized controlled trial of metformin versus vitamin $E$ or prescriptive diet in nonalcoholic fatty liver disease. $\mathrm{Am}$ J Gastroenterol 2005, 100:1082-1090.

63.• Belfort R, Harrison SA, Brown K, et al.: A Placebo-controlled trial of pioglitazone in subjects with nonalcoholic steatohepatitis. N Engl J Med 2006, 355:2297-2307.

Randomized placebo-controlled trial of 6 months that compared the effect of diet in combination with pioglitazone to diet and placebo in 55 individuals with NAFLD. It demonstrated a significant reduction in liver fat content and a significant increase in hepatic insulin sensitivity.

64. Ding X, Saxena NK, Lin S, et al.: Exendin-4, a glucagonlike protein-1 (GLP-1) receptor agonist, reverses hepatic steatosis in ob/ob mice. Hepatology 2006, 43:173-181.

65. Tushuizen ME, Bunck MC, Pouwels PJ, et al.: Incretin mimetics as a novel therapeutic option for hepatic steatosis. Liver Int 2006, 26:1015-1017.

66. Curioni C, Andre C: Rimonabant for overweight or obesity. Cochrane Database Syst Rev 2006, (4):CD006162.

67. Teixeira-Clerc F, Julien B, Grenard P, et al.: CB1 cannabinoid receptor antagonism: a new strategy for the treatment of liver fibrosis. Nat Med 2006, 12:671-676. 